\title{
Bicyclic Graphs with the Second-Maximum and Third-Maximum Degree Resistance Distance
}

\author{
Wenjie Ning $\mathbb{D},{ }^{1}$ Kun Wang $(\mathbb{D})^{2}$ and Hassan Raza $\mathbb{D}^{3}$ \\ ${ }^{1}$ College of Science, China University of Petroleum (East China), Qingdao 266580, China \\ ${ }^{2}$ College of Mathematics and Systems Science, Shandong University of Science and Technology, Qingdao 266590, China \\ ${ }^{3}$ Business School, University of Shanghai for Science and Technology, Shanghai 200093, China
}

Correspondence should be addressed to Kun Wang; wangkun26@163.com

Received 7 August 2021; Accepted 14 September 2021; Published 10 November 2021

Academic Editor: Francisco Balibrea

Copyright (@) 2021 Wenjie Ning et al. This is an open access article distributed under the Creative Commons Attribution License, which permits unrestricted use, distribution, and reproduction in any medium, provided the original work is properly cited.

Let $G=(V, E)$ be a connected graph. The resistance distance between two vertices $u$ and $v$ in $G$, denoted by $R_{G}(u, v)$, is the effective resistance between them if each edge of $G$ is assumed to be a unit resistor. The degree resistance distance of $G$ is defined as $D_{R}(G)=\sum_{\{u, v\} \subseteq V(G)}\left(d_{G}(u)+d_{G}(v)\right) R_{G}(u, v)$, where $d_{G}(u)$ is the degree of a vertex $u$ in $G$ and $R_{G}(u, v)$ is the resistance distance between $u$ and $v$ in $G$. A bicyclic graph is a connected graph $G=(V, E)$ with $|E|=|V|+1$. This paper completely characterizes the graphs with the second-maximum and third-maximum degree resistance distance among all bicyclic graphs with $n \geq 6$ vertices.

\section{Introduction}

All graphs considered in this paper are simple and undirected. Let $G=(V, E)$ be a graph with $n$ vertices and $m$ edges. Let $N_{G}(v)$ be the set of vertices adjacent to $v$ in $G$. The degree of $v$ in $G$, denoted by $d_{G}(v)$, is equal to $\left|N_{G}(v)\right|$. Denote the minimum degree of vertices in $G$ by $\delta(G)$. A vertex of degree one is called a pendant vertex, and the edge incident with a pendant vertex is called a pendant edge. The distance between two vertices $u$ and $v$ of $G$, denoted by $d_{G}(u, v)$ or $d(u, v)$, is the length of a shortest path connecting $u$ and $v$ in $G$. For a subset $S$ of $V$, denote by $G[S]$, the subgraph induced by $S$ and $G-S$ the graph $G[V(G) \backslash S]$. We use $G-v$ instead of $G-\{v\}$ if $S=\{v\}$ for simplicity. Let $P_{n}$ and $C_{n}$ be the path and the cycle graphs on $n$ vertices, respectively.

A topological index or a graph-theoretic index is a real number related to a graph. Topological indices of molecular graphs are one of the oldest and most widely used descriptors in quantitative structure-activity relationships $[1,2]$. One of the most exhaustively studied $[3,4]$ topological indices is the Wiener index. The Wiener index was introduced in 1947 [5] and defined as $W(G)=\sum_{\{u, v\} \subseteq V(G)} d_{G}(u, v)$. It is well correlated with many physical and chemical properties of organic molecules and chemical compounds.

Based on the electrical network theory, Klein and Randić [6] proposed a novel distance function called resistance distance in 1993. They treated a graph $G$ as an electric network by considering each edge of $G$ as a unit resistor. Then, the resistance distance between two vertices $u$ and $v$ in $G$, denoted by $R_{G}(u, v)$, is defined as the effective resistance between them. Klein and Randić [6] also proved that $R_{G}(u, v) \leq d_{G}(u, v)$, with equality if and only if there is a unique path connecting $u$ and $v$ in $G$. In recent years, this new type of distance between vertices in a graph has attracted prominent attention in mathematics and chemistry [6-11].

Similar to the Wiener index, the Kirchhoff index of a graph $G$ is defined as

$$
K f(G)=\sum_{\{u, v\} \subseteq V(G)} R_{G}(u, v) .
$$

This invariant has wide applications in electric circuit, physical interpretations, chemistry, and graph theory [12-16]. 
In 2012, Gutman et al. [17] introduced the concept of the degree resistance distance defined as

$$
D_{R}(G)=\sum_{\{u, v\} \subseteq V(G)}\left(d_{G}(u)+d_{G}(v)\right) R_{G}(u, v) .
$$

Palacios called it as additive degree-Kirchhoff index in [18]. In [17], Gutman et al. [17] presented some properties of $D_{R}(G)$ and characterized the unicyclic graphs with the minimum and second-minimum $D_{R}(G)$. Later, the unicyclic graphs with the maximum and second-maximum $D_{R}$-value were considered in $[19,20]$. In $[21,22]$, the cactus graphs with the minimum, the second-minimum, and the thirdminimum $D_{R}$-values were also completely characterized. Recently, the bicyclic graphs with maximum and minimum $D_{R}$-values were determined in $[23,24]$, respectively.

A bicyclic graph $G=(V, E)$ is a connected graph such that $|E|=|V|+1$. The kernel of $G$, denoted by $G$, is the unique bicyclic subgraph of $G$ with no pendant vertices. Any bicyclic graph $G$ is obtained from its kernel $G$ by attaching trees to some vertices in $G$. Given a family of graphs $\mathscr{G}$, the graphs with the maximum and second maximum values of topological indices among $\mathscr{G}$ are examined widely, see in [25-29]. Motivated by this, in this paper, we determine the graphs with the second-maximum and third-maximum degree resistance distance among all bicyclic graphs with $n \geq 6$ vertices.

\section{Preliminaries}

Let $\mathscr{B}_{n}$ be the set of bicyclic graphs of order $n, \mathscr{B}_{n}^{\infty}$ be the set of bicyclic graphs of order $n$ with exactly two cycles, and $\mathscr{B}_{n}^{\theta}=\mathscr{B}_{n} \backslash \mathscr{B}_{n}^{\infty}$. Let $B(p, q)$ be obtained from two vertexdisjoint cycles $C_{p}$ and $C_{q}$ by identifying a vertex $u \in V\left(C_{p}\right)$ and a vertex $v \in V\left(C_{q}\right), B(p, l, q)$ be obtained from two vertex-disjoint cycles $C_{p}$ and $C_{q}$ by connecting a vertex $u \in V\left(C_{p}\right)$ and a vertex $v \in V\left(C_{q}\right)$ by a path $u v_{1} v_{2} \ldots v_{l-1} v$ of length $l(l \geq 1)$, and $B\left(P_{r}, P_{s}, P_{t}\right)$ be the union of three internally disjoint paths $P_{r}, P_{s}$, and $P_{t}$, respectively, with common end vertices, where $r, s, t \geq 2$ and at most one of them is 2 .

Let $G$ be a graph and $v$ be a vertex in $G$. Define $K f_{v}(G)=$ $\sum_{u \in V(G)} R_{G}(u, v)$ and $D_{v}(G)=\sum_{u \in V(G)} d_{G}(u) R_{G}(u, v)$.

We present a few lemmas which will be employed later to establish our main results.

Lemma 1 (see [13]). Let $G$ be a connected graph with a pendant vertex $v$ with its unique neighbor $w$. Then, $K f_{v}(G)=K f_{w}(G-v)+n-1$.

Lemma 2 (see [13]). Let $G$ be a bicyclic graph of order $n$ and $v \in V(G)$. Then, $K f_{v}(G) \leq n^{2} / 2-n / 2-15 / 4$. Moreover, if $d_{G}(v) \geq 2$, then $K f_{v}(G) \leq n^{2} / 2-3 n / 2+1 / 3$.

The following remark can be obtained from the proof of Lemma 2.

Remark 1. Let $G$ be a graph in $\mathscr{B}_{n}^{\infty}$ and $v \in V(G)$. Then, $K f_{v}(G) \leq n^{2} / 2-n / 2-6$.
Lemma 3 (see [17]). Let $G$ be a connected graph with a cut vertex $v$ such that $G_{1}$ and $G_{2}$ are two connected subgraphs of $G$ having $v$ as the only common vertex and $V\left(G_{1}\right) \bigcup V\left(G_{2}\right)=V(G)$. Let $n_{1}=\left|V\left(G_{1}\right)\right|, n_{2}=\left|V\left(G_{2}\right)\right|$, $m_{1}=\left|E\left(G_{1}\right)\right|$, and $m_{2}=\left|E\left(G_{2}\right)\right|$. Then, $D_{R}(G)=D_{R}\left(G_{1}\right)+$ $D_{R}\left(G_{2}\right)+2 m_{2} K f_{v}\left(G_{1}\right)+2 m_{1} K f_{v}\left(G_{2}\right)+\left(n_{2}-1\right) D_{v}\left(G_{1}\right)+$ $\left(n_{1}-1\right) D_{v}\left(G_{2}\right)$.

Lemma 4 (see [17]). Let $C_{k}$ be a cycle with length $k$ and $v \in C_{k}$. Then, $K f\left(C_{k}\right)=\left(k^{3}-k\right) / 12, D_{R}\left(C_{k}\right)=\left(k^{3}-k\right) / 3$, $K f_{v}\left(C_{k}\right)=\left(k^{2}-1\right) / 6$, and $D_{v}\left(C_{k}\right)=\left(k^{2}-1\right) / 3$.

Lemma 5 (see [23]). Let $H$ be a connected graph of order $h>2$ and $C_{k}$ be a cycle of order $k \geq 4$. Let $F$ be the graph of order $k$ obtained from $C_{3}$ by attaching one pendant path of order $k-3$ to one vertex of $C_{3}$. Further suppose $G_{1}$ is the graph obtained from $H$ and $C_{k}$ by identifying one vertex in $H$ and one vertex in $C_{k} ; G_{2}$ is the graph obtained from $H$ and $F$ by identifying one vertex in $H$ and the pendant vertex in $F$. Then, we have $D_{R}\left(G_{1}\right)<D_{R}\left(G_{2}\right)$.

By an argument similar to that of Lemma 5, we easily get the following result.

Lemma 6. Let $G$ be a connected graph of order $n>2$ and $C_{k}$ be a cycle of order $k \geq 5$. Let $F$ be obtained by identifying $a$ pendant vertex of $P_{k-3}$ with any vertex of $C_{4}$. Suppose $G_{1}$ is the graph obtained from $G$ and $C_{k}$ by identifying one vertex in $G$ and one vertex in $C_{k} ; G_{2}$ is obtained from $G$ and $F$ by identifying one vertex in $G$ and the pendant vertex in $F$. Then, $D_{R}\left(G_{1}\right)<D_{R}\left(G_{2}\right)$.

In [23], Du and $\mathrm{Tu}$ characterized the unique bicyclic graph with maximum degree resistance distance. They also presented two significant lemmas in [23].

Theorem 1 (see [23]). Let $G$ be a bicyclic graph of order $n \geq 6$; then, $D_{R}(G) \leq 2 n^{3} / 3+n^{2}-19 n+88 / 3$, with equality if and only if $G \cong B(3, n-5,3)$.

Lemma 7 (see [23]). Let $G$ be a bicyclic graph of order $n$ and $v \in V(G)$. Then, $D_{v}(G) \leq n^{2}+2 n-73 / 4$.

Lemma 8 (see [23]). Let $G$ be a bicyclic graph of order $n$, vbe a pendant vertex of $G$, and $w$ be its neighbor. Then, $D_{R}(G)=$ $D_{R}(G-v)+D_{w}(G-v)+2 K f_{w}(G-v)+3 n$.

\section{Bicyclic Graphs with the Second-Maximum Degree Resistance Distance}

In this section, we will determine the bicyclic graphs with the second-maximum degree resistance distance.

Suppose $n \geq 6$. Let $B(3, n-5,3)$ be obtained from two 3 cycles $v_{1} v_{2} v_{3} v_{1}$ and $v_{n-2} v_{n-1} v_{n} v_{n-2}$ by connecting $v_{3}$ and $v_{n-2}$ by a path $v_{3} v_{4} \cdots v_{n-3} v_{n-2}$. Define $G_{n}^{1}=B(3, n-5,3)-$ $v_{n-1} v_{n}+v_{n-1} v_{n-3}$ and $G_{n}^{2, i}=G_{n}^{1}-v_{n-2} v_{n}+v_{i} v_{n}$, where $3 \leq i \leq n-3$. Let $G_{n}^{3}\left(G_{n}^{5}\right)$ be obtained from a 4 -cycle $C_{4}=$ $v_{1} v_{2} v_{3} v_{4} v_{1}$ and a path $P=v_{5} \ldots v_{n}$ by adding the edges $v_{1} v_{3}$ $\left(v_{2} v_{4}\right.$, resp. $)$ and $v_{4} v_{5}$. Let $G_{n}^{4} \cong B(4, n-6,3)$ be obtained 
from a 4-cycle $v_{1} v_{2} v_{3} v_{4} v_{1}$ and a 3-cycle $v_{n-2} v_{n-1} v_{n} v_{n-2}$ by connecting $v_{4}$ and $v_{n-2}$ by a path $v_{4} v_{5} \ldots v_{n-3} v_{n-2}$ (see Figure 1). Then, we have the following lemma.

Lemma 9. Let $G_{n}^{1}, G_{n}^{2, i}, G_{n}^{3}, G_{n}^{4}$, and $G_{n}^{5}$ be defined as above. Then, $\quad D_{R}\left(G_{n}^{1}\right)=2 / 3 n^{3}+n^{2}-79 / 3 n+56, D_{R}\left(G_{n}^{2, i}\right)$
$=2 / 3 n^{3}+n^{2}-17 n+4 i^{2}-4 n i+88 / 3, \quad D_{R}\left(G_{n}^{3}\right)=2 / 3 n^{3}+$ $n^{2}-293 / 12 n+117 / 2, \quad D_{R}\left(G_{n}^{4}\right)=2 / 3 n^{3}+n^{2}-82 / 3 n+$ $167 / 3$, and $D_{R}\left(G_{n}^{5}\right)=2 / 3 n^{3}+n^{2}-163 / 6 n+139 / 2$.

Proof. By Lemma 8 and Theorem 1, we easily obtain

$$
\begin{aligned}
& D_{R}\left(G_{n}^{1}\right)=D_{R}\left(G_{n}^{1}-v_{n}\right)+D_{v_{n-2}}\left(G_{n}^{1}-v_{n}\right)+2 K f_{v_{n-2}}\left(G_{n}^{1}-v_{n}\right)+3 n \\
& =\left[\frac{2}{3}(n-1)^{3}+(n-1)^{2}-19(n-1)+\frac{88}{3}\right] \\
& +\left[2 \cdot \frac{2}{3}+3 \cdot \frac{2}{3}+2 \cdot\left(\frac{2}{3}+1\right)+2 \cdot\left(\frac{2}{3}+2\right)+\cdots+2 \cdot\left(\frac{2}{3}+n-7\right)+3 \cdot\left(\frac{2}{3}+n-6\right)\right. \\
& \left.+2 \cdot\left(\frac{4}{3}+n-6\right)+2 \cdot\left(\frac{4}{3}+n-6\right)\right]+2 \cdot\left[\frac{2}{3}+\frac{2}{3}+\left(\frac{2}{3}+1\right)\right. \\
& \left.+\left(\frac{2}{3}+2\right)+\cdots+\left(\frac{2}{3}+n-6\right)+2\left(\frac{4}{3}+n-6\right)\right]+3 n \\
& =\left[\frac{2}{3}(n-1)^{3}+(n-1)^{2}-19(n-1)+\frac{88}{3}\right] \\
& +\left(n^{2}-\frac{14}{3} n+\frac{4}{3}\right)+2 \cdot\left(\frac{n^{2}}{2}-\frac{17}{6} n+3\right)+3 n \\
& =\frac{2}{3} n^{3}+n^{2}-\frac{79}{3} n+56 \\
& D_{R}\left(G_{n}^{2, i}\right)=D_{R}\left(G_{n}^{2, i}-v_{n}\right)+D_{v_{i}}\left(G_{n}^{2, i}-v_{n}\right)+2 K f_{v_{i}}\left(G_{n}^{2, i}-v_{n}\right)+3 n \\
& =\left[\frac{2}{3}(n-1)^{3}+(n-1)^{2}-19(n-1)+\frac{88}{3}\right]+[2 \cdot 1+2 \cdot 2 \\
& +\cdots+2 \cdot(i-4)+3 \cdot(i-3)+4 \cdot\left(i-3+\frac{2}{3}\right)+2 \cdot 1+2 \cdot 2 \\
& \left.+\cdots+2 \cdot(n-4-i)+3 \cdot(n-3-i)+4 \cdot\left(n-3-i+\frac{2}{3}\right)\right] \\
& +2 \cdot\left[1+2+\cdots+(i-3)+2 \cdot\left(i-3+\frac{2}{3}\right)+1+2+\cdots\right. \\
& \left.+(n-3-i)+2 \cdot\left(n-3-i+\frac{2}{3}\right)\right]+3 n \\
& =\left(\frac{2}{3} n^{3}-n^{2}-19 n+\frac{146}{3}\right)+\left(n^{2}+2 i^{2}-2 n i-\frac{38}{3}\right) \\
& +2 \cdot \frac{3 n^{2}-3 n+6 i^{2}-6 n i-20}{6}+3 n \\
& =\frac{2}{3} n^{3}+n^{2}-17 n+4 i^{2}-4 n i+\frac{88}{3} .
\end{aligned}
$$

Let $H=G_{n}^{3}\left[\left\{v_{1}, v_{2}, v_{3}, v_{4}\right\}\right]$. By Lemma 3, 


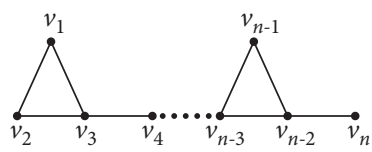

$G_{n}^{1}$
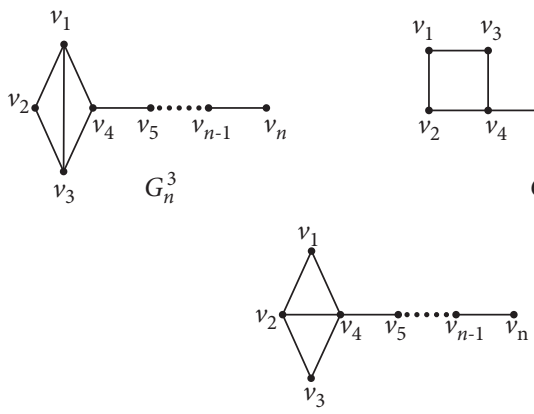

$G_{n}^{5}$

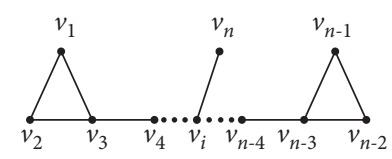

$G_{n}^{2, i}$

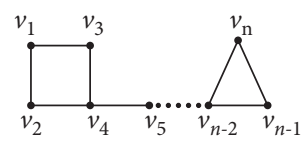

$G_{n}^{4}$

Figure 1: Graphs $G_{n}^{1}, G_{n}^{2, i}, G_{n}^{3}, G_{n}^{4}$, and $G_{n}^{5}$.

$$
\begin{aligned}
D_{R}\left(G_{n}^{3}\right)= & D_{R}(H)+D_{R}\left(P_{n-3}\right)+2(n-4) K f_{v_{4}}(H)+10 K f_{v_{4}}\left(P_{n-3}\right) \\
& +(n-4) D_{v_{4}}(H)+3 D_{v_{4}}\left(P_{n-3}\right) \\
= & \frac{39}{2}+\left[\frac{2}{3}(n-3)^{3}-(n-3)^{2}+\frac{1}{3}(n-3)\right]+2 \cdot(n-4) \cdot \frac{9}{4} \\
& +10 \cdot \frac{(n-3)(n-4)}{2}+(n-4) \cdot \frac{23}{4}+3 \cdot(n-4)^{2} \\
= & \frac{2}{3} n^{3}+n^{2}-\frac{293}{12} n+\frac{117}{2} .
\end{aligned}
$$

Let $F=G_{n}^{4}-\left\{v_{n-1}, v_{n}\right\}$. By Lemmas 3 and 6 ,

$$
\begin{aligned}
D_{R}\left(G_{n}^{4}\right)= & D_{R}\left(C_{3}\right)+D_{R}(F)+2(n-2) K f_{v_{n-2}}\left(C_{3}\right)+6 K f_{v_{n-2}}(F) \\
& +(n-3) D_{v_{n-2}}\left(C_{3}\right)+2 D_{v_{n-2}}(F) \\
= & 8+\left[\frac{2}{3}(n-2)^{3}-\frac{53}{3}(n-2)+48\right]+\frac{8}{3}(n-2)+6\left[\frac{(n-2)^{2}}{2}\right. \\
& \left.-\frac{n-2}{2}-\frac{7}{2}\right]+\frac{8}{3}(n-3)+2\left[(n-2)^{2}-11\right] \\
& =\frac{2}{3} n^{3}+n^{2}-\frac{82}{3} n+\frac{167}{3} .
\end{aligned}
$$

Let $S=G_{n}^{5}\left[\left\{v_{1}, v_{2}, v_{3}, v_{4}\right\}\right]$. By Lemma 3, 


$$
\begin{aligned}
D_{R}\left(G_{n}^{5}\right)= & D_{R}(S)+D_{R}\left(P_{n-3}\right)+2(n-4) K f_{v_{4}}(S)+10 K f_{v_{4}}\left(P_{n-3}\right) \\
& +(n-4) D_{v_{4}}(S)+3 D_{v_{4}}\left(P_{n-3}\right) \\
= & \frac{39}{2}+\left[\frac{2}{3}(n-4)^{3}+(n-4)^{2}+\frac{1}{3}(n-4)\right]+2(n-4) \cdot \frac{7}{4} \\
& +10 \cdot \frac{(n-3)(n-4)}{2}+4(n-4)+3(n-4)^{2} \\
= & \frac{2}{3} n^{3}+n^{2}-\frac{163}{6} n+\frac{139}{2} .
\end{aligned}
$$

Theorem 2. Suppose $G$ is a graph in $\mathscr{B}_{n}^{\infty}$ with $G \not \equiv B(3, n-$ $5,3)$ and $n \geq 6$. Then, $D_{R}(G) \leq 2 / 3 n^{3}+n^{2}-79 / 3 n+56$, with equality if and only if $G \cong G_{n}^{1}$, where $G_{n}^{1}$ is defined as in Lemma 9 .

Proof. It is easy to verify that, for any graph $G$ in $\mathscr{B}_{6}^{\infty}$ with $G ¥ B(3,1,3), D_{R}(G) \leq 78=2 / 3 \cdot 6^{3}+6^{2}-79 / 3 \cdot 6+56$, with equality if and only if $G \cong G_{6}^{1}$.

Now, we assume $n \geq 7$ and consider the following two cases.

Case $1 \delta(G)=1$ : let $v$ be a pendant vertex in $G$. If $G-v \cong B(3, n-6,3)$, then either $G \cong G_{n}^{1}$, or $G \cong G_{n}^{2, i}$, where $G_{n}^{1}$ and $G \cong G_{n}^{2, i}$ are defined as in Lemma 9. By Lemma 9,

$$
\begin{aligned}
D_{R}\left(G_{n}^{2, i}\right) & =\frac{2}{3} n^{3}+n^{2}-17 n+4 i^{2}-4 n i+\frac{88}{3} \\
& \leq \frac{2}{3} n^{3}+n^{2}-17 n+4 \cdot 3^{2}-4 n \cdot 3+\frac{88}{3} \\
& <\frac{2}{3} n^{3}+n^{2}-\frac{79}{3} n+56 .
\end{aligned}
$$

If $G-v \not B(3, n-6,3)$, we prove it by induction on $n$. Let $w$ be the neighbor of $v$. By the inductive hypothesis, Remark 1, and Lemmas 7-9

$$
\begin{aligned}
D_{R}(G)= & D_{R}(G-v)+D_{w}(G-v)+2 K f_{w}(G-v)+3 n \\
\leq & \frac{2}{3}(n-1)^{3}+(n-1)^{2}-\frac{79}{3}(n-1) \\
& +56+\left[(n-1)^{2}+2(n-1)\right. \\
& \left.-\frac{73}{4}\right]+2\left(\frac{(n-1)^{2}}{2}-\frac{n-1}{2}-6\right)+3 n \\
= & \frac{2}{3} n^{3}+n^{2}-\frac{79}{3} n+\frac{641}{12} \\
< & \frac{2}{3} n^{3}+n^{2}-\frac{79}{3} n+56 .
\end{aligned}
$$

Case $2(\delta(G) \geq 2)$ : in this case, $G$ is of the form $B(p, q)$ or $B(p, l, q)$. By Lemmas 5 and 6 , we have $D_{R}(G) \leq D_{R}\left(G_{n}^{4}\right)$, with equality if and only if $G \cong G_{n}^{4}$.
Note that $D_{R}\left(G_{n}^{4}\right)<D_{R}\left(G_{n}^{1}\right)$ by Lemma 9. Therefore, the proof is complete.

Theorem 3. Suppose $G$ is a graph of ordern $\geq 4$ in $\mathscr{B}_{n}^{\theta}$. Then, $D_{R}(G) \leq 2 / 3 n^{3}+n^{2}-293 / 12 n+117 / 2$, with equality if and only if $G \cong G_{n}^{3}$, where $G_{n}^{3}$ is defined in Lemma 9 .

Proof. It is easy to verify that the only graph in $\mathscr{B}_{4}^{\theta}$ is $G_{4}^{3}$ and $D_{R}\left(G_{4}^{3}\right)=2 / 3 \cdot 4^{3}+4^{2}-293 / 12 \cdot 4+117 / 2$. We assume $n \geq 5$ next, and consider the following two cases.

Case $1(\delta(G)=1)$ : let $v$ be a pendant vertex in $G$ and $w$ be the neighbor of $v$. We prove it by induction on $n$. By the inductive hypothesis, Lemma 2, and Lemmas 7-9,

$$
\begin{aligned}
D_{R}(G)= & D_{R}(G-v)+D_{w}(G-v)+2 K f_{w}(G-v)+3 n \\
\leq & \frac{2}{3}(n-1)^{3}+(n-1)^{2}-\frac{293}{12}(n-1) \\
& +\frac{117}{2}+\left[(n-1)^{2}+2(n-1)\right. \\
& \left.-\frac{73}{4}\right]+2 \cdot\left(\frac{(n-1)^{2}}{2}-\frac{n-1}{2}-\frac{15}{4}\right)+3 n \\
= & \frac{2}{3} n^{3}+n^{2}-\frac{293}{12} n+\frac{117}{2} .
\end{aligned}
$$

The equality $D_{R}(G-v)=2 / 3 n^{3}+n^{2}-293 / 12 n+117 / 2$ holds if and only if $D_{R}(G-v)=2 / 3(n-1)^{3}+(n-1)^{2}$ $-293 / 12(n-1)+117 / 2, \quad D_{w}(G-v)=(n-1)^{2}+2$. $(n-1) \quad-73 / 4$, and $K f_{w}(G-v)=(n-1)^{2} / 2-(n$ $-1) / 2-15 / 4=n^{2} / 2-3 / 2 n-11 / 4$. By the inductive hypothesis, $G-v \cong G_{n-1}^{3}$, which is obtained from a 4-cycle $C_{4}=v_{1} v_{2} v_{3} v_{4} v_{1}$ and a path $P=v_{5} \ldots v_{n-1}$ by adding the edges $v_{1} v_{3}$ and $v_{4} v_{5}$. We show that $w=v_{n-1}$, i.e., $G \cong G_{n}^{3}$. By direct calculation, we have $K f_{v_{n-1}}\left(G_{n-1}^{3}\right)=$ $n^{2} / 2-3 / 2 n-11 / 4, K f_{v_{1}}\left(G_{n-1}^{3}\right)=K f_{v_{3}}\left(G_{n-1}^{3}\right)^{3}=n^{2} / 2-$ $31 / 8 n+69 / 8<n^{2} / 2-3 / 2 n-11 / 4$, and $K f_{v_{2}}\left(G_{n-1}^{3}\right)=$ $n^{2} / 2-7 / 2 n+29 / 4<n^{2} / 2-3 / 2 n-11 / 4$. Obviously, $K f_{u}\left(G_{n-1}^{3}\right)<K f_{v_{n-1}}\left(G_{n-1}^{3}\right) \quad$ if $\quad u \in V\left(G_{n-1}^{3}\right) \backslash\left\{v_{1}, v_{2}\right.$, $\left.v_{3}, v_{n-1}\right\}$. Therefore, $w=v_{n-1}$, i.e., $G \cong G_{n}^{3}$. 
Case $2(\delta(G) \geq 2)$ : then, $G$ is of the form $B\left(P_{k}, P_{l}, P_{m}\right)$. Suppose $x$ and $y$ are the only two vertices of degree 3 . Since $K f(G) \leq 1 / 8 n^{3}$ (see [13]), we have

$$
\begin{aligned}
D_{R}(G) & =\sum_{\{u, v\} \subseteq V(G)}(d(u)+d(v)) R(u, v) \\
& =4 K f(G)+K f_{x}(G)+K f_{y}(G) \\
& \leq 4 \cdot \frac{1}{8} n^{3}+2 \cdot\left(\frac{1}{2} n^{2}-\frac{3}{2} n+\frac{1}{3}\right)(\text { by Lemma } 2) \\
& =\frac{1}{2} n^{3}+n^{2}-3 n+\frac{2}{3} .
\end{aligned}
$$

If $n \geq 10$, then $1 / 2 n^{3}+n^{2}-3 n+2 / 3<2 / 3 n^{3}+n^{2}-$ $293 / 12 n+117 / 2$. For any graph $G \cong B\left(P_{k}, P_{l}, P_{m}\right)$ when $n=5,6,7,8,9$, we have calculated $D_{R}(G)$ and found that $D_{R}(G)<2 / 3 n^{3}+n^{2}-293 / 12 n+117 / 2$.

Combining Theorems 1-3, we can obtain the first main result of our paper.
Theorem 4. Suppose $G$ is a bicyclic graph of order $n \geq 6$ with $G \not B(3, n-5,3)$. Then, $D_{R}(G) \leq 2 / 3 n^{3}+n^{2}-293 / 12 n+$ $117 / 2$, with equality if and only if $G \cong G_{n}^{3}$, where $G_{n}^{3}$ is defined as in Lemma 9.

\section{Bicyclic Graphs with the Third-Maximum Degree Resistance Distance}

In this section, we will determine the bicyclic graphs with the third-maximum degree resistance distance.

Lemma 10. Let $G_{n}^{3, i}$ be obtained from a 4-cycle $C_{4}=$ $v_{1} v_{2} v_{3} v_{4} v_{1}$, a path $P=v_{5} \ldots v_{n-1}$ and an isolated vertex $v_{n}$ by adding the edges $v_{1} v_{3}, v_{4} v_{5}$, and $v_{i} v_{n}$, where $1 \leq i \leq n-2$ and $n \geq 6$. Then, $D_{R}\left(G_{n}^{3,1}\right)=D_{R}\left(G_{n}^{3,3}\right)=2 / 3 n^{3}+n^{2} \quad-455 /$ $12 n+493 / 4, D_{R}\left(G_{n}^{3,2}\right)=2 / 3 n^{3}+n^{2}-437 / 12 n+237 / 2, D_{R}$ $\left(G_{n}^{3,4}\right)=2 / 3 n^{3}+n^{2}-485 / 12 n+277 / 2$, and $D_{R}\left(G_{n}^{3, i}\right)=$ $2 / 3 n^{3}+n^{2}-293 / 12 n-4 n i+4 i^{2}+4 i+117 / 2$, for $5 \leq i$ $\leq n-2$.

Proof. By Lemmas 8 and 9, we easily obtain

$$
\begin{aligned}
& D_{R}\left(G_{n}^{3,1}\right)=D_{R}\left(G_{n}^{3,3}\right)=D_{R}\left(G_{n-1}^{3}\right)+D_{v_{1}}\left(G_{n-1}^{3}\right)+2 K f_{v_{1}}\left(G_{n-1}^{3}\right)+3 n \\
& =\left[\frac{2}{3}(n-1)^{3}+(n-1)^{2}-\frac{293}{12}(n-1)+\frac{117}{2}\right] \\
& +2 \cdot \frac{5}{8}+3 \cdot \frac{5}{8}+3 \cdot \frac{1}{2}+2 \cdot\left(\frac{5}{8}+1\right)+\cdots+2 \cdot\left(\frac{5}{8}+n-6\right)+\left(n-5+\frac{5}{8}\right) \\
& +2 \cdot\left[\frac{5}{8}+\frac{5}{8}+\frac{1}{2}+\left(\frac{5}{8}+1\right)+\cdots+\left(\frac{5}{8}+n-5\right)\right]+3 n \\
& =\left[\frac{2}{3}(n-1)^{3}+(n-1)^{2}-\frac{293}{12}(n-1)+\frac{117}{2}\right]+\left(n^{2}-\frac{35}{4} n+\frac{91}{4}\right) \\
& +\left(n^{2}-\frac{31}{4} n+\frac{69}{4}\right)+3 n=\frac{2}{3} n^{3}+n^{2}-\frac{455}{12} n+\frac{493}{4}, \\
& D_{R}\left(G_{n}^{3,2}\right)=D_{R}\left(G_{n-1}^{3}\right)+D_{v_{2}}\left(G_{n-1}^{3}\right)+2 K f_{v_{2}}\left(G_{n-1}^{3}\right)+3 n \\
& =\left[\frac{2}{3}(n-1)^{3}+(n-1)^{2}-\frac{293}{12}(n-1)+\frac{117}{2}\right] \\
& +3 \cdot \frac{5}{8}+3 \cdot \frac{5}{8}+3 \cdot 1+2 \cdot 2+\cdots+2 \cdot(n-5)+(n-4)+2 \cdot\left(\frac{5}{8}+\frac{5}{8}+1\right. \\
& +\cdots+n-4)+3 n
\end{aligned}
$$




$$
\begin{aligned}
= & {\left[\frac{2}{3}(n-1)^{3}+(n-1)^{2}-\frac{293}{12}(n-1)+\frac{117}{2}\right] } \\
& +\left(n^{2}-8 n+\frac{83}{4}\right) \\
& +\left(n^{2}-7 n+\frac{29}{2}\right)+3 \\
= & {\left[\frac{2}{3} n^{3}+n^{2}-\frac{437}{12} n+\frac{237}{2},\right.} \\
& D_{R}\left(G_{n}^{3,4}\right)=D_{R}\left(G_{n-1}^{3}\right)+D_{v_{4}}\left(G_{n-1}^{3}\right)+2 K f_{v_{4}}\left(G_{n-1}^{3}\right)+3 n \\
= & {\left[\frac{2}{3}(n-1)^{3}+(n-1)^{2}-\frac{293}{12}(n-1)+\frac{117}{2}\right] } \\
& {\left[+2 \cdot 3 \cdot \frac{5}{8}+2 \cdot 1+2 \cdot 1+2 \cdot 2+\cdots+2 \cdot(n-6)+(n-5)\right]+2 \cdot\left(2 \cdot \frac{5}{8}+1+1\right.} \\
& +\cdots+n-5+3 n=\left[\frac{2}{3}(n-1)^{3}+(n-1)^{2}-\frac{293}{12}(n-1)+\frac{117}{2}\right]+\left(n^{2}-10 n+\frac{123}{4}\right) \\
& +\left(n^{2}-9 n+\frac{49}{2}\right)+3 n=\frac{2}{3} n^{3}+n^{2}-\frac{485}{12} n+\frac{277}{2},
\end{aligned}
$$

and for $5 \leq i \leq n-2$,

$$
\begin{aligned}
D_{R}\left(G_{n}^{3, i}\right)= & D_{R}\left(G_{n-1}^{3}\right)+D_{v_{i}}\left(G_{n-1}^{3}\right)+2 K f_{v_{i}}\left(G_{n-1}^{3}\right)+3 n \\
= & {\left[\frac{2}{3}(n-1)^{3}+(n-1)^{2}-\frac{293}{12}(n-1)+\frac{117}{2}\right] } \\
& +2 \cdot 1+2 \cdot 2+\cdots \\
& +2 \cdot(n-2-i)+(n-1-i)+2 \cdot[1+2 \cdot 2+\cdots+2 \cdot(i-5) \\
& +3 \cdot(i-4)+2 \cdot 3 \cdot\left(i-4+\frac{5}{8}\right)+2 \cdot(i-3)+2 \cdot 1+2+\cdots \\
& \left.+(n-1-i)+1+2+\cdots+i-3+2 \cdot\left(i-4+\frac{5}{8}\right)\right]+3 n \\
= & {\left[\frac{2}{3}(n-1)^{3}+(n-1)^{2}-\frac{293}{12}(n-1)+\frac{117}{2}\right] } \\
& +\left(n^{2}-2 n i-2 n+2 i^{2}+4 i-\frac{69}{4}+n^{2}-2 n i-n+2 i^{2}-\frac{15}{2}\right)+3 n \\
= & \frac{2}{3} n^{3}+n^{2}-\frac{293}{12} n-4 n i+4 i^{2}+4 i+\frac{117}{2} .
\end{aligned}
$$

Proposition 1. Suppose $G \neq G_{n}^{3}$ is a bicyclic graph of ordern $\geq 5$ and $v \in V(G)$, where $G_{n}^{3}$ is defined as in Lemma 9. Then, $K f_{v}(G) \leq n^{2} / 2-n / 2-17 / 4$.
Proof. It is not hard to verify that, for any bicyclic graph $G \neq G_{5}^{3}$ of order 5 and $v \in V(G), K f_{v}(G) \leq 5^{2} / 2-5 / 2-17 / 4$. Thus, we assume $n \geq 6$ in the following cases. 
Case $1(d(v)=1)$ : let $w$ be the neighbor of $v$. Suppose $G-v \cong G_{n-1}^{3}$, where $G_{n-1}^{3}$ is obtained from a 4cycle $C_{4}=v_{1} v_{2} v_{3} v_{4} v_{1}$ and a path $P=v_{5} \ldots v_{n-1}$ by adding the edges $v_{1} v_{3}$ and $v_{4} v_{5}$. Then, $w \neq v_{n-1}$ since $G ¥ G_{n}^{3}$. By Lemma 1 ,

$$
\begin{aligned}
K f_{v}(G) & =K f_{w}(G-v)+n-1 \\
& \leq \max \left\{K f_{v_{n-2}}\left(G_{n-1}^{3}\right), K f_{v_{2}}\left(G_{n-1}^{3}\right)\right\}+n-1 \\
& =\max \left\{\frac{n^{2}}{2}-\frac{5}{2} n+\frac{1}{4}, \frac{n^{2}}{2}-\frac{7}{2} n+\frac{29}{4}\right\}+n-1 \\
& =\max \left\{\frac{n^{2}}{2}-\frac{3}{2} n-\frac{3}{4}, \frac{n^{2}}{2}-\frac{5}{2} n+\frac{25}{4}\right\} \\
& <\frac{n^{2}}{2}-\frac{n}{2}-\frac{17}{4} .
\end{aligned}
$$

If $G-v \neq G_{n-1}^{3}$, we shall prove it by induction on $n$. By the inductive hypothesis, $K f_{v}(G)=K f_{w}(G-v)+$ $n-1 \leq(n-1)^{2} / 2-(n-1) / 2-17 / 4+n-1=n^{2} / 2-$ $n / 2-17 / 4$.

Case 2: $d(v) \geq 2$.

By Lemma 2, $K f_{v}(G) \leq n^{2} / 2-3 n / 2+1 / 3<n^{2} / 2-$ $n / 2-17 / 4$

Lemma 11 (see [23]). Let $G$ be a bicyclic graph of order $n, v$ be a pendant vertex of $G$, and $w$ be its neighbor. Then, $D_{v}(G)=D_{w}(G-v)+2 n+1$.

Proposition 2. Let $G ¥ G_{n}^{3}$ be a graph in $\mathscr{B}_{n}^{\theta}$ of order $n \geq 5$ and $v \in V(G)$, where $G_{n}^{3}$ is defined as in Lemma 9. Then, $D_{v}(G) \leq n^{2}+2 n-20$.

Proof. It is easy to verify that for any graph $G \in \mathscr{B}_{5}^{\theta}$ with $G \not G_{5}^{3}$ and $v \in V(G), D_{v}(G) \leq 15=5^{2}+2 \cdot 5-20$. Thus, we assume $n \geq 6$ in the following cases.

Case $1\left(d_{G}(v)=1\right)$ : let $w$ be the neighbor of $v$.

Suppose $G-v \cong G_{n-1}^{3}$, where $G_{n-1}^{3}$ is obtained from a 4cycle $C_{4}=v_{1} v_{2} v_{3} v_{4} v_{1}$ and a path $P=v_{5} \ldots v_{n-1}$ by adding the edges $v_{1} v_{3}$ and $v_{4} v_{5}$. Then, $w \neq v_{n-1}$ since $G ¥ G_{n}^{3}$. Moreover, $D_{v}(G)=D_{w}\left(G_{n-1}^{3}\right)+2 n+1$ by Lemma 11. By direct calculation, we get $D_{v_{1}}$ $\left(G_{n-1}^{3}\right)=D_{v_{3}}\left(G_{n-1}^{3}\right)=n^{2}-35 / 4 n+91 / 4, \quad D_{v_{2}}\left(G_{n-1}^{3}\right) \stackrel{v_{1}}{=}$ $n^{2}-8 n+83 / 4, D_{v_{4}}\left(G_{n-1}^{3}\right)=n^{2}-10 n+123 / 4$, and

$$
\begin{aligned}
D_{v_{i}}\left(G_{n-1}^{3}\right) & =n^{2}-2 n+2 i^{2}+(4-2 n) i-\frac{69}{4} \\
& \leq n^{2}-2 n+2(n-2)^{2}+(4-2 n)(n-2)-\frac{69}{4} \\
& =n^{2}-2 n-\frac{69}{4}\left(=D_{v_{n-2}}\left(G_{n-1}^{3}\right)\right),
\end{aligned}
$$

if $5 \leq i \leq n-2$. Thus, $D_{w}\left(G_{n-1}^{3}\right) \leq D_{v_{n-2}}\left(G_{n-1}^{3}\right)$ and $D_{v}(G) \leq D_{v_{n-2}}\left(G_{n-1}^{3}\right)+2 n+1=n^{2}-65 / 4<n^{2}+2 n-20$. If $G-v \neq G_{n-1}^{3}$, we prove it by induction on $n$. By the inductive hypothesis, $D_{v}(G)=D_{w}(G)+2 n+1 \leq$ $(n-1)^{2}+2(n-1)-20+2 n+1=n^{2}+2 n-20$.

Case $2: d_{G}(v) \geq 2$.

Subcase 1: $v$ is not contained by any cycle of $G$.

By the same argument as that of Case 2 of Lemma 2.6 in [23], we can construct a series of bicyclic graphs $G_{1}, G_{2}, \ldots, G_{k-1}$ in $\mathscr{B}_{n}^{\theta}$ such that $D_{v}(G)<D_{v}$ $\left(G_{1}\right)<\cdots<D_{v}\left(G_{k-1}\right)$ and $v$ is a pendant vertex in $G_{k-1}$, where $k=d_{G}(v) \geq 2$.

Suppose $G_{k-1} \cong G_{n}^{3}$. Then, $G_{k-1}$ is obtained from a 4cycle $C_{4}=v_{1} v_{2} v_{3} v_{4} v_{1}$ and a path $P=v_{5} \cdots v_{n-1} v$ by adding the edges $v_{1} v_{3}$ and $v_{4} v_{5}$. By the transformation from $G_{k-2}$ to $G_{k-1}$, we can conclude that $G_{k-2}=G_{k-1}-v_{n-2} v_{n-1}+v_{n-2}$ v, i.e., $G_{k-2} \cong G_{k-1}$. Note that $D_{v}\left(G_{k-2}\right)=n^{2}-73 / 4$. We have $D_{v}(G) \leq D_{v}$ $\left(G_{k-2}\right)<n^{2}+2 n-20$.

If $G_{k-1} \not G_{n}^{3}$, then, by Case $1, D_{v}(G)<D_{v}\left(G_{k-1}\right) \leq$ $n^{2}+2 n-20$.

Subcase 2: $v$ is in a cycle of $G$.

Let $\hat{G}$ be the kernel of $G$. By Claims 1 and 2 of Lemma 2.6 in [23], we can construct a graph $G^{\prime \prime}$ in $\mathscr{B}_{n}^{\theta}$ having $G$ as its kernel and $D_{v}(G) \leq D_{v}\left(G^{\prime \prime}\right)$. Moreover, $G^{\prime \prime}$ is obtained from $G$ by attaching a pendant path to the vertex $u$, where $u$ is a vertex of $G$ such that $R_{G}(u, v)=\max \wedge R_{G}(w, v)$.

Suppose $G^{\prime \prime}$ has only two vertices of degree three, say $w_{1}$ and $w_{2}$. Without loss of generality, we assume that $v \neq w_{1}$, and $v \neq w_{2}$. Then, by Lemma 2,

$$
\begin{aligned}
D_{v}\left(G^{\prime \prime}\right) & =3\left(R_{G^{\prime \prime}}\left(w_{1}, v\right)+R_{G^{\prime \prime}}\left(w_{2}, v\right)\right)+\sum_{w \neq w_{1}, w_{2}} 2 R_{G^{\prime \prime}}(w, v) \\
& =R_{G^{\prime \prime}}\left(w_{1}, v\right)+R_{G^{\prime \prime}}\left(w_{2}, v\right)+2 K f_{v}\left(G^{\prime \prime}\right) \\
& <d_{G^{\prime \prime}}\left(w_{1}, v\right)+d_{G^{\prime \prime}}\left(w_{2}, v\right)+2 K f_{v}\left(G^{\prime \prime}\right) \\
& \leq n+2\left(\frac{n^{2}}{2}-\frac{3}{2} n+\frac{1}{3}\right) \\
& <n^{2}+2 n-20 .
\end{aligned}
$$

Suppose $G^{\prime \prime}$ has exactly three vertices of degree three, say $w_{1}, w_{2}$, and $w_{3}$. Let $w_{4}$ be the pendant vertex of $G^{\prime \prime}$. Without loss of generality, we assume that $v \neq w_{1}, w_{2}, w_{3}$. Then, by Lemma 2,

$$
\begin{aligned}
D_{v}\left(G^{\prime \prime}\right)= & 3\left(R_{G^{\prime \prime}}\left(w_{1}, v\right)+R_{G^{\prime \prime}}\left(w_{2}, v\right)+R_{G^{\prime \prime}}\left(w_{3}, v\right)\right)+R_{G^{\prime \prime}}\left(w_{4}, v\right) \\
& +\sum_{w \neq w_{1}, w_{2}, w_{3}, w_{4}} 2 R_{G^{\prime \prime}}(w, v) \\
< & R_{G^{\prime \prime}}\left(w_{1}, v\right)+R_{G^{\prime \prime}}\left(w_{2}, v\right)+R_{G^{\prime \prime}}\left(w_{3}, v\right)+2 K f_{v}\left(G^{\prime \prime}\right) \\
< & d_{G^{\prime \prime}}\left(w_{1}, v\right)+d_{G^{\prime \prime}}\left(w_{2}, v\right)+d_{G^{\prime \prime}}\left(w_{3}, v\right)+2 K f_{v}\left(G^{\prime \prime}\right)
\end{aligned}
$$




$$
\begin{aligned}
& \leq \frac{3(n-1)}{2}+2 \cdot\left(\frac{n^{2}}{2}-\frac{3}{2} n+\frac{1}{3}\right) \\
& \leq n^{2}+2 n-20 .
\end{aligned}
$$

Suppose $G^{\prime \prime}$ has a vertex of degree four, say $w_{1}$, and a vertex of degree three, say $w_{2}$. Let $w_{3}$ be the pendant vertex of $G^{\prime \prime}$. Without loss of generality, we assume that $v \neq w_{1}, w_{2}$. Then, by Lemma 2,

$$
\begin{aligned}
D_{v}\left(G^{\prime \prime}\right)= & 4 R_{G^{\prime \prime}}\left(w_{1}, v\right)+3 R_{G^{\prime \prime}}\left(w_{2}, v\right) \\
& +R_{G^{\prime \prime}}\left(w_{3}, v\right)+\sum_{w \neq w_{1}, w_{2}, w_{3}} 2 R_{G^{\prime \prime}}(w, v) \\
< & 2 R_{G^{\prime \prime}}\left(w_{1}, v\right)+R_{G^{\prime \prime}}\left(w_{2}, v\right)+2 K f_{v}\left(G^{\prime \prime}\right) \\
< & 2 d_{G^{\prime \prime}}\left(w_{1}, v\right)+d_{G^{\prime \prime}}\left(w_{2}, v\right)+2 K f_{v}\left(G^{\prime \prime}\right) \\
\leq & \frac{3(n-1)}{2}+2 \cdot\left(\frac{n^{2}}{2}-\frac{3}{2} n+\frac{1}{3}\right) \\
\leq & n^{2}+2 n-20,
\end{aligned}
$$

which completes the proof.

Theorem 5. Suppose $G$ is a graph of order $n \geq 5$ in $\mathscr{B}_{n}^{\theta} \backslash\left\{G_{n}^{3}\right\}$. Then, $D_{R}(G) \leq 2 / 3 n^{3}+n^{2}-163 / 6 n+139 / 2$, with equality if and only if $G \cong G_{n}^{5}$, where $G_{n}^{3}$ and $G_{n}^{5}$ are defined as in Lemma 9.

Proof. It is not hard to verify that, for any graph $G$ in $\mathscr{B}_{5}^{\theta} \backslash\left\{G_{5}^{3}\right\}, \quad D_{R}(G) \leq 42=2 / 3 \cdot 5^{3}+5^{2}-163 / 6 \cdot 5+139 / 2$, with equality if and only if $G \cong G_{5}^{5}$.

We assume that $n \geq 6$, and consider the following two cases.

Case 1: $\delta(G)=1$.

Let $v_{n}$ be a pendant vertex of $G$. Suppose $G-v_{n} \cong G_{n-1}^{3}$, where $G_{n-1}^{3}$ is obtained from a 4 -cycle $C_{4}=v_{1} v_{2} v_{3} v_{4} v_{1}$, and a path $P=v_{5} \cdots v_{n-1}$ by adding the edges $v_{1} v_{3}$ and $v_{4} v_{5}$. Then, $G \cong G_{n}^{3, i}$, where $1 \leq i \leq n-2$, and $G_{n}^{3, i}$ is defined in the Lemmas 10. By Lemma 10,

$$
\begin{aligned}
D_{R}\left(G_{n}^{3,1}\right)= & D_{R}\left(G_{n}^{3,3}\right)=\frac{2}{3} n^{3}+n^{2}-\frac{455}{12} n+\frac{493}{4}<\frac{2}{3} n^{3}+n^{2}-\frac{163}{6} n+\frac{139}{2}, \\
D_{R}\left(G_{n}^{3,2}\right)= & \frac{2}{3} n^{3}+n^{2}-\frac{437}{12} n+\frac{237}{2}<\frac{2}{3} n^{3}+n^{2}-\frac{163}{6} n+\frac{139}{2}, \\
D_{R}\left(G_{n}^{3,4}\right)= & \frac{2}{3} n^{3}+n^{2}-\frac{485}{12} n+\frac{277}{2}<\frac{2}{3} n^{3}+n^{2}-\frac{163}{6} n+\frac{139}{2}, \\
D_{R}\left(G_{n}^{3, i}\right)= & \frac{2}{3} n^{3}+n^{2}-\frac{293}{12} n-4 n i+4 i^{2}+4 i+\frac{117}{2} \\
\leq & \frac{2}{3} n^{3}+n^{2}-\frac{293}{12} n-4 n(n-2)+4(n-2)^{2}+4(n-2)+\frac{117}{2} \\
& =\frac{2}{3} n^{3}+n^{2}-\frac{341}{12} n+\frac{133}{2} \\
& <\frac{2}{3} n^{3}+n^{2}-\frac{163}{6} n+\frac{139}{2},
\end{aligned}
$$

for $5 \leq i \leq n-2$.

If $G-v_{n} \not G_{n-1}^{3}$, we prove it by induction on $n$. Let $w$ be the neighbor of $v_{n}$. By the inductive hypothesis, Lemma 8, and Propositions 1 and 2, 


$$
\begin{aligned}
D_{R}(G)= & D_{R}\left(G-v_{n}\right)+D_{w}\left(G-v_{n}\right)+2 K f_{w}\left(G-v_{n}\right)+3 n \\
\leq & {\left[\frac{2}{3}(n-1)^{3}+(n-1)^{2}-\frac{163}{6}(n-1)\right.} \\
& \left.+\frac{139}{2}\right]+(n-1)^{2}+2(n-1) \\
& -20+2 \cdot\left(\frac{(n-1)^{2}}{2}-\frac{n-1}{2}-\frac{17}{4}\right)+3 n \\
= & \frac{2}{3} n^{3}+n^{2}-\frac{163}{6} n+\frac{139}{2} .
\end{aligned}
$$

The equality $D_{R}(G)=2 / 3 n^{3}+n^{2}-163 / 6 n+139 / 2$ holds if and only if $D_{R}\left(G-v_{n}\right)=2 / 3(n-1)^{3}+(n-1)^{2}$ $-163 / 6(n-1)+139 / 2, D_{w}\left(G-v_{n}\right)=(n-1)^{2}+2(n-$ 1) -20 , and $K f_{w}\left(G-v_{n}\right)=(n-1)^{2} / 2-(n-1) / 2-$ $17 / 4=n^{2} / 2-3 / 2 n-13 / 4$. By the inductive hypothesis, $G-v_{n} \cong G_{n-1}^{5}$, where $G_{n-1}^{5}$ is obtained from a 4 -cycle $C_{4}=v_{1} v_{2} v_{3} v_{4} v_{1}$ and a path $P=v_{5} \ldots v_{n-1}$ by adding the edges $v_{2} v_{4}$ and $v_{4} v_{5}$. We show that $w=v_{n-1}$, i.e., $G \cong G_{n}^{5}$.

By direct calculation, we have $K f_{v_{n-1}}\left(G_{n-1}^{5}\right)=$ $n^{2} / 2-3 / 2 n-13 / 4, K f_{v_{2}}\left(G_{n-1}^{5}\right)=n^{2} / 2-4 n+37 / 4<n^{2}$ $/ 2-3 / 2 n-13 / 4$, and $K f_{v_{1}}\left(G_{n-1}^{5}\right)=K f_{v_{3}}\left(G_{n-1}^{5}\right)=$ $n^{2} / 2-31 / 8 n+73 / 8<n^{2} / 2 \quad-3 / 2 n-13 / 4$. Obviously, $K f_{v}\left(G_{n-1}^{5}\right)<K f_{v_{n-1}}\left(G_{n-1}^{5}\right) \quad$ if $\quad v \in V\left(G_{n-1}^{5}\right) \backslash\left\{v_{1}, v_{2}\right.$, $\left.v_{3}, v_{n-1}\right\}$. Therefore, $w=v_{n-1}$, i.e., $G \cong G_{n}^{5}$.

Case 2: $\delta(G) \geq 2$.

By a similar argument to that of Case 2 in Theorem 3, we obtain

$$
D_{R}(G) \leq \frac{1}{2} n^{3}+n^{2}-3 n+\frac{2}{3} .
$$

If $n \geq 11$, then $1 / 2 n^{3}+n^{2}-3 n+2 / 3<2 / 3 n^{3}+n^{2}-$ $163 / 6 n+139 / 2$. For any graph of the form $B\left(P_{k}, P_{l}, P_{m}\right)$ when $n=6,7,8,9,10$, we have calculated $D_{R}(G)$ and found that $D_{R}(G) \leq 2 / 3 n^{3}+n^{2}-163 / 6 n+139 / 2$.

From Theorems 2 and 4, we obtain the following result.

Theorem 6. Let $G_{n}^{1}$ and $G_{n}^{5}$ be defined as in Lemma 9. Then, among all bicyclic graphs of order $n$,

(i) If $6 \leq n \leq 16$, the graph $G_{n}^{5}$ is the unique graph with the third-maximum degree resistance distance of value $2 / 3 n^{3}+n^{2}-163 / 6 n+139 / 2$

(ii) If $n \geq 17$, the graph $G_{n}^{1}$ is the unique graph with the third-maximum degree resistance distance of value $2 / 3 n^{3}+n^{2}-79 / 3 n+56$

\section{Conclusion}

As a molecular structure descriptor, the Wiener index is one of the widely employed topological indices, as it is well correlated with many physical and chemical properties of a variety of classes of chemical compounds. A weighted version of the Wiener index is the degree resistance distance. In this paper, we characterize the graphs with the secondmaximum and third-maximum degree resistance distance among all bicyclic graphs with fixed order. Furthermore, we present an open problem.

Problem 1. Characterize the tricyclic graphs of order $n$ with the maximum and second-maximum degree resistance distance.

\section{Data Availability}

All the proofs and exemplary data of this study are included within the article.

\section{Conflicts of Interest}

The authors declare that they have no conflicts of interest.

\section{Acknowledgments}

The research of the first author was supported by National Natural Science Foundation of China (no. 11801568).

\section{References}

[1] R. Gozalbes, J. Doucet, and F. Derouin, "Application of topological descriptors in QSAR and drug design: history and new trends," Current Drug Targets-Infectious Disorders, vol. 2, pp. 93-102, 2002.

[2] O. Ivanciuc, "QSAR comparative study of Wiener descriptor for weighted molecular graphs," Journal of Chemical Information and Computer Sciences, vol. 40, pp. 1412-1422, 2000.

[3] K. C. Das, I. Gutman, and M. J. Nadjafi-Arani, "Relations between distance-based and degree-based topological indices," Applied Mathematics and Computation, vol. 270, pp. 142-147, 2015.

[4] K. Xu, M. Liu, K. C. Das, I. Gutman, and B. Furtula, "A survey on graphs extremal with respect to distance-based topological indices," MATCH Communications in Mathematical and in Computer Chemistry, vol. 71, pp. 461-508, 2014.

[5] H. Wiener, "Structural determination of paraffin boiling points," Journal of the American Chemical Society, vol. 69, pp. 17-20, 1947.

[6] D. J. Klein and M. Randić, "Resistance distance," Journal of Mathematical Chemistry, vol. 12, pp. 81-95, 1993.

[7] D. Babić, D. J. Klein, I. Lukovits, S. Nikolić, and N. Trinajstić, "Resistance-distance matrix: a computational algorithm and its application," International Journal of Quantum Chemistry, vol. 90, pp. 166-176, 2002.

[8] R. B. Bapat, I. Gutman, and W. Xiao, "A simple method for computing resistance distance," Zeitschrift für Naturforschung, vol. 58a, pp. 494-498, 2003.

[9] X. Gao, Y. Luo, and W. Liu, "Resistance distances and the Kirchhoff index in Cayley graphs," Discrete Applied Mathematics, vol. 159, pp. 2050-2057, 2011.

[10] Y. Yang and D. J. Klein, "A recursion formula for resistance distances and its applications," Discrete Applied Mathematics, vol. 161, pp. 2702-2715, 2013.

[11] Y. Yang and H. Zhang, "Some rules on resistance distance with applications," Journal of Physics A: Mathematical and Theoretical, vol. 41, Article ID 445203, 2008. 
[12] C. Arauz, "The Kirchhoff indexes of some composite networks," Discrete Applied Mathematics, vol. 160, pp. 14291440, 2012.

[13] L. Feng, G. Yu, K. Xu, and Z. Jiang, "A note on the Kirchhoff index of bicyclic graphs," Ars Combinatoria, vol. 114, pp. 33-40, 2014.

[14] J. L. Palacios and J. M. Renom, "Another look at the degreeKirchhoff index," International Journal of Quantum Chemistry, vol. 111, pp. 3453-3455, 2011.

[15] B. Zhou and N. Trinajstić, "Mathematical properties of molecular descriptors based on distances," Croatica Chemica Acta, vol. 83, pp. 227-242, 2010.

[16] A. D. Maden, A. S. Cevik, I. N. Cangul, and K. C. Das, "On the Kirchhoff matrix, a new Kirchhoff index and the Kirchhoff energy," Journal of Inequalities and Applications, vol. 337, 2013.

[17] I. Gutman, L. Feng, and G. Yu, "Degree resistance distance of unicyclic graphs," Trans. Comb.vol. 1, no. 2, pp. 27-40, 2012.

[18] J. L. Palacios, "Upper and lower bounds for the additive degree-Kirchhoff index," MATCH Communications in Mathematical and in Computer Chemistry, vol. 70, pp. 651655, 2013.

[19] S. Chen, Q. Chen, X. Cai, and Z. Guo, "Maximal degree resistance distance of unicyclic graphs," MATCH Communications in Mathematical and in Computer Chemistry, vol. 75, pp. 157-168, 2016.

[20] J. Tu, J. Du, and G. Su, "The unicyclic graphs with maximum degree resistance distance," Applied Mathematics and Computation, vol. 268, pp. 859-864, 2015.

[21] J. Du, G. Su, J. Tu, and I. Gutman, "The degree resistance distance of cacti," Discrete Applied Mathematics, vol. 188, pp. 16-24, 2015.

[22] J. Liu, W. Wang, Y. Zhang, and X. Pan, "On degree resistance distance of cacti," Discrete Applied Mathematics, vol. 203, pp. 217-225, 2016.

[23] J. Du and J. Tu, "Bicyclic graphs with maximum degree resistance distance," Filomat, vol. 30, pp. 1625-1632, 2016.

[24] J. Liu, S. Zhang, X. Pan, S. Wang, and S. Hayat, "Bicyclic graphs with extremal degree resistance distance," arXiv: 1606.01281v1, 2016.

[25] Z. Du and B. Zhou, "On sum-connectivity index of bicyclic graphs," Bulletin of the Malaysian Mathematical Sciences Society, vol. 35, pp. 101-117, 2012.

[26] R. Xing, B. Zhou, and F. Dong, "On atom-bond connectivity index of connected graphs," Discrete Applied Mathematics, vol. 159, pp. 1617-1630, 2011.

[27] J. Li and J. Zhang, "On the second Zagreb eccentricity indices of graphs," Applied Mathematics and Computation, vol. 352, pp. 180-187, 2019.

[28] J. Fei and J. Tu, "Complete characterization of bicyclic graphs with the maximum and second-maximum degree Kirchhoff index," Applied Mathematics and Computation, vol. 330, pp. 118-124, 2018.

[29] L. Zhong and Q. Cui, "The harmonic index for unicyclic graphs with given girth," Filomat, vol. 29, pp. 673-686, 2015. 